\title{
Essentials of Human Disease in Dentistry
}

Author: Mark Greenwood

Publisher:Wiley-Blackwell, Hoboken, NJ

07030, USA

Language: English

ISBN: 978-1-119-25185-9

Edition: 2/e

Publish Year: 2018

Pages: 376, illustrated

Price: $\$ 83.25$

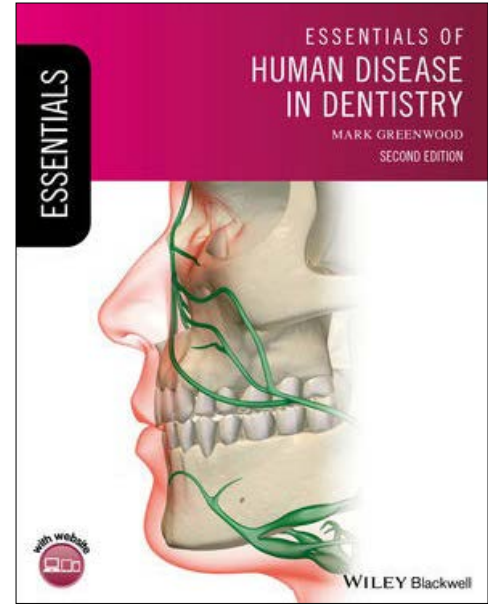

Marian-Vladimir

Constantinescu

$\mathrm{DDS}, \mathrm{MSC}, \mathrm{PhD}$

Holistic Dental \& Medical Institute

of Bucharest-ROPOSTURO,

Bucharest, Romania

e-mail:

dr.vladimir.constantinescu@gmail.com

In current practice, a dentist has the opportunity to provide specialized care to patients with various general diseases.

Dr. Mark Greenwood, author of the second edition of Essentials of Human Disease in Dentistry, improved the first edition, namely Textbook of Human Disease in Dentistry, adding aspects related to general medicine and surgery, pharmacy, pathology and microbiology.

The book is an accessible guide on human disease for dentistry divided in 21 chapters, accompanied by an appendix and an index.

First of all, it presents the areas that need to be covered in the medical history, and then, the book presents the clinical observations and the examination of the hands, face, cardiovascular system, respiratory system, and vital signs.

The potential consequences of tissue injury, inflammation and anti-inflammatory drugs are then described. The author presents an overview of infection and infection control, methods of infection control, clinical examples of bacterial, viral and fungal infections and their management, and then an overview of abnormal immune response, abnormalities in the immune response, immunodeficiency, autoimmunity, and allergy.

The area of major cardiovascular disorders is accompanied by an overview of the main drugs used to treat risks from bacteremia-producing dental procedures and their impact on the practice of dentistry.

The impact of respiratory disorders, gastrointestinal disorders, kidney disorders, neurology disorders and muscular-skeletal disorders on dentistry is further explained.

Major dermatological disorders and their oral manifestations, major endocrine glands disorders, dental aspects of endocrine disorders, and implications for patient management are covered in the following chapters.

In describing pain and anxiety control we become acquainted with local anesthesia and adverse effects, vasoconstrictors, commonly used analgesics, drugs used in conscious sedation, adverse drug reactions, and their interactions.

The author also covers various aspects related to general oncology and oral complications of malignancy, child development, infections in childhood, medicine for the elderly patients, common psychiatric disorders, general aspects of hematology and hemostasis, and management of medical emergencies in dental practice. Each chapter is accompany by suggestive schemes and figures, which can be accessed on the Internet, on the companion website of the publisher, multiple choice questions, together with relevant references on the topics covered. This second edition of Essentials of Human Disease in Dentistry is an invaluable resource for undergraduate dentistry students, for dentists preparing for the MFDS exam, and for all dental practitioners.

DOI: http://www.stomaeduj.com 10.25241/stomaeduj.2019.6(3).bookreview.6

The Books Review is drafted in the reviewer's sole wording and illustrates his opinions. 